\title{
Common Fixed Point Theorems for Two Mappings in Complete $b$-Metric Spaces
}

\author{
Lili Chen, Xin Xia, Yanfeng Zhao * and Xin Liu
}

College of Mathematics and Systems Science, Shandong University of Science and Technology, Qingdao 266590, China; cll2119@sdust.edu.cn (L.C.); x2244533231@126.com (X.X.); 1xin2020@126.com (X.L.)

* Correspondence: zhaoyf1101@sdust.edu.cn

\begin{abstract}
Our paper is devoted to the issue of the existence and uniqueness of common fixed points for two mappings in complete $b$-metric spaces by virtue of the new functions $F$ and $\theta$, respectively. Moreover, two specific examples to indicate the validity of our results are also given. Eventually, the generalized forms of Jungck fixed point theorem in the above spaces is investigated. Different from related literature, the conditions that the function $F$ needs to satisfy are weakened, and $F$ only needs to be non-decreasing in this paper. To some extent, our conclusions and methods improve the results of previous literature.
\end{abstract}

Keywords: existence and uniqueness; common fixed point theorems; $b$-metric spaces

MSC: 47H09; 47H10

check for updates

Citation: Chen, L.; Xia, X.; Zhao, Y.; Liu, X. Common Fixed Point Theorems for Two Mappings in Complete $b$-Metric Spaces. Fractal Fract. 2022, 6, 103. https://doi.org/ $10.3390 /$ fractalfract6020103

Academic Editor: Sotiris K. Ntouyas, Bashir Ahmad and Jessada Tariboon

Received: 20 January 2022

Accepted: 8 February 2022

Published: 11 February 2022

Publisher's Note: MDPI stays neutral with regard to jurisdictional claims in published maps and institutional affiliations.

Copyright: (c) 2022 by the authors. Licensee MDPI, Basel, Switzerland. This article is an open access article distributed under the terms and conditions of the Creative Commons Attribution (CC BY) license (https:// creativecommons.org/licenses/by/ $4.0 /)$.

\section{Introduction}

The conceptual framework of $b$-metric spaces, as a meaningful generalization of metric spaces, was first formally proposed by Czerwik [1] who discussed the convergence of measurable functions and also established the Banach contraction principle in $b$-metric spaces. Subsequently, the Banach contraction principle plays an important role in $b$-metric spaces, and it is one of the most valid tools in the research fields of nonlinear analysis and its applications. In fact, it is extensively regarded as the beginnings of metric fixed point theory.

Thereafter, many scholars have focused on fixed point problems in the tendency of the generalization of $b$-metric spaces. To be specific, Samet [2] fully certified that the class of $(\alpha, \psi)$-type contractions contains a good deal of contraction-type operators, and the fixed points of the operators can be obtained in virtue of the Picard iteration. Mohanta [3] discussed the existence and uniqueness of common fixed points for mappings defined on a $b$-metric space endowed with a graph. In [4], the authors gave some common fixed point results for a pair of self-mappings that satisfy $g$-generalized weakly contractive conditions in a $b$-metric space endowed with an amorphous binary relation.

In [5], an interesting generalization of the Banach contraction principle was shown by introducing the notion of $F$-contractions, which as a new type of contraction, have been applied to obtain fixed point results for single-valued mappings and multi-valued mappings in $b$-metric spaces. In [6], Cosentinoet et al. introduced the notion of HardyRogers-type $F$-contractions as a generalization of $F$-contractions in complete metric spaces. Moreover, a number of consequences related to $F$-contractions and their extensions have been obtained, for details please see [7-17]. Suzuki [18] investigated fixed point theorems for set-valued $F$-contractions in complete $b$-metric spaces and also proposed a fixed point theorem for single-valued $F$-contractions in complete $b$-metric spaces. Moreover, Mirmostafaee et al. [19] established a set-valued version of Suzuki's fixed point theorem in complete $b$-metric spaces. Jang [20] presented Hardy-Rogers-type and Reich-type common fixed point theorems in complete metric spaces which generalize and unify previously 
known results. Recently, the existence and uniqueness of fixed points for $F$-contractions in complete Branciari $b$-metric spaces were considered in [21]. The theory of set-valued mappings has been established to extend the framework of fixed point theorems in $b$-metric spaces and has applications in control theory, convex optimization, differential inclusions, and economics.

Motivated by the above-mentioned discussions, we mainly study the existence and uniqueness of common fixed points for two mappings in complete $b$-metric spaces by virtue of the new functions $F$ and $\theta$, respectively. Furthermore, we present two specific instances to show the availability of our results. In the specific proof process, we discuss and deal with various cases in detail. Compared with the previous results, we weaken the conditions of the function $F$, which only needs to be non-decreasing. Hence, to some extent, our conclusions and methods improve the results of previous literature.

\section{Preliminaries}

We begin with some auxiliary lemmas and basic definitions in this section. Let $\mathcal{N}$ be the set of all positive integers and $\mathcal{R}$ be the set of all real numbers respectively.

Definition 1 ([22]). Let $V$ be a non-empty set and consider $s \geq 1$ be a given real number. $A$ function $d_{b}: V \times V \rightarrow[0, \infty)$ is a b-metric if the following conditions are satisfied for every $c_{1}, c_{2}, c_{3} \in V:$

$\left(a_{1}\right) d_{b}\left(c_{1}, c_{2}\right)=0$ if and only if $c_{1}=c_{2}$

$\left(a_{2}\right) d_{b}\left(c_{1}, c_{2}\right)=d_{b}\left(c_{2}, c_{1}\right)$

$\left(a_{3}\right) d_{b}\left(c_{1}, c_{2}\right) \leq s\left[d_{b}\left(c_{1}, c_{3}\right)+d_{b}\left(c_{3}, c_{2}\right)\right]$.

In this case, the pair $\left(V, d_{b}\right)$ is called a $b$-metric space with constant $s \geq 1$.

Definition 2 ([5]). A function $\mathbb{F}:(0,+\infty) \rightarrow \mathbb{R}$ belongs to $\mathcal{F}$ if the following conditions are satisfied:

$\left(F_{1}\right) \mathbb{F}$ is strictly decreasing;

$\left(F_{2}\right)$ for each sequence $\left\{\alpha_{n}\right\}\left(\alpha_{n}>0\right), \lim _{n \rightarrow+\infty} \alpha_{n}=0$ if and only if $\lim _{n \rightarrow+\infty} \mathbb{F}\left(\alpha_{n}\right)=-\infty$;

$\left(F_{3}\right)$ there exists $k \in(0,1)$ such that $\lim _{x \rightarrow 0^{+}} x^{k} \mathbb{F}(x)=0$.

Definition 3 ([5]). Let $(V, d)$ be a metric space and $P: V \rightarrow V$ be a mapping. Assume that there exist $\mathbb{F} \in \mathcal{F}$ and $\tau>0$ such that for all $x, y \in V$, the inequality $d(P x, P y)>0$ implies $\tau+\mathbb{F}(\operatorname{sd}(P x, P y)) \leq \mathbb{F}(d(x, y))$, then $P$ is called an F-contraction.

Example 1 ([5]). Let $(V, d)$ be a b-metric space and $F:(0, \infty) \rightarrow \mathbb{R}$ be defined by $F(\kappa)=\ln \kappa$. Then $F$ satisfies (F1)-(F3). Each mapping $P: V \rightarrow V$ satisfying the inequality of Definition 3 is an F-contraction such that

$$
d_{b}(P x, P y) \leq e^{-\tau} d_{b}(x, y), \text { for all } x, y \in V, P x \neq P y .
$$

In the case of $P x=P y$, the inequality also holds, we obtain that every Banach contraction is an F-contraction.

Example 2 ([5]). Consider $F(\kappa)=\ln \kappa+\kappa, \kappa>0$. F satisfies (F1)-(F3) and the inequality of Definition 3 implies

$$
\frac{d_{b}(P x, P y)}{d_{b}(x, y)} e^{d_{b}(P x, P y)-d_{b}(x, y)} \leq e^{-\tau}, \text { for all } x, y \in V, P x \neq P y .
$$

Let $L, J: V \rightarrow V$ be two mappings defined on a metric space $(V, d)$. If there exists $u \in V$ such that $u=L(u)$, then $u$ is said to be a fixed point of $L$. Moreover, if $u=L(u)=$ $J(u)$, then $u$ is said to be a common fixed point of the mappings $L$ and $J$. 
Theorem 1 ([23]). Let L be a continuous self-map on a complete metric space $(V, d)$. Then L has a fixed point if and only if there exist a constant $\varphi \in[0,1)$ and a map $J: V \rightarrow V$ which commutes with $L$ and the following conditions hold

$$
J(V) \subseteq L(V) \text { and } d(J(u), J(t)) \leq \varphi d(L(u), L(t)) \text { for all } u, t \in V .
$$

Indeed, L and J have a unique common fixed point if the above conditions hold.

Theorem 2 ([24]). Let $L: V \rightarrow V$ be a self-map on a complete metric space $(V, d)$. If there exist the constants $\varphi, \omega, \mu, \eta, \lambda \in[0,1)$ with $\varphi+\omega+\mu+\eta+\lambda<1$ such that for all $u, t \in V$,

$$
d(L(u), L(t)) \leq \varphi d(u, L(u))+\omega d(t, L(t))+\mu d(u, L(t))+\eta d(t, L(u))+\lambda d(u, t),
$$

then L has a unique fixed point.

Before giving the main results, we first show a useful lemma.

Lemma 1. Let $L$ and $J$ be self-mappings on a b-metric space $\left(V, d_{b}\right)$ with $s \geq 1$. Suppose that there exist $\varphi, \omega, \mu, \eta, \lambda \in[0,1)$ with $\varphi+\omega+s \mu+s \eta+\lambda<1$ such that

$$
\begin{aligned}
d_{b}\left(L\left(u_{1}\right), J\left(u_{2}\right)\right) \leq & \varphi d_{b}\left(u_{1}, L\left(u_{1}\right)\right)+\omega d_{b}\left(u_{2}, J\left(u_{2}\right)\right)+\mu d_{b}\left(u_{1}, J\left(u_{2}\right)\right) \\
& +\eta d_{b}\left(u_{2}, L\left(u_{1}\right)\right)+\lambda d_{b}\left(u_{1}, u_{2}\right)
\end{aligned}
$$

for all $u_{1}, u_{2} \in V$. Then

$$
\begin{gathered}
d_{b}(L(u), J(L(u))) \leq \frac{\varphi+s \mu+\lambda}{1-s \mu-\omega} d_{b}(u, L(u)), \\
d_{b}(J(t), L(J(t))) \leq \frac{\omega+s \eta+\lambda}{1-s \eta-\varphi} d_{b}(t, J(t)),
\end{gathered}
$$

for all $u \in V$ and $t \in L(V)$.

Proof. Let $u \in V$. It is not difficult to see that

$$
\begin{aligned}
d_{b}(L(u), J(L(u))) \leq & \varphi d_{b}(u, L(u))+\omega d_{b}(L(u), J(L(u)))+\mu d_{b}(u, J(L(u))) \\
& +\lambda d_{b}(u, L(u))
\end{aligned}
$$

By using condition $\left(a_{3}\right)$ of Definition 1 and adding $-s \mu d_{b}(u, L(u))$ on both sides of $(1)$, we obtain

$$
\begin{aligned}
& d_{b}(L(u), J(L(u)))-s \mu d_{b}(u, L(u)) \\
& \leq \varphi d_{b}(u, L(u))+\omega d_{b}(L(u), J(L(u)))+\mu d_{b}(u, J(L(u))) \\
& \quad-s \mu d_{b}(u, L(u))+\lambda d_{b}(u, L(u)) \\
& \leq \varphi d_{b}(u, L(u))+\omega d_{b}(L(u), J(L(u)))+s \mu d_{b}(L(u), J(L(u)))+\lambda d_{b}(u, L(u)) .
\end{aligned}
$$

Thus

$$
d_{b}(L(u), J(L(u))) \leq \frac{\varphi+s \mu+\lambda}{1-s \mu-\omega} d_{b}(u, L(u)) .
$$

Let $t \in L(V)$. Notice that

$$
\begin{aligned}
d_{b}(J(t), L(J(t))) & =d_{b}(L(J(t)), J(t)) \\
& \leq \varphi d_{b}(J(t), L(J(t)))+\omega d_{b}(t, J(t))+\eta d_{b}(t, L(J(t)))+\lambda d_{b}(J(t), t) .
\end{aligned}
$$


By adding $-s \eta d_{b}(J(t), L(J(t)))$ on both sides of (2), we have

$$
\begin{aligned}
d_{b}(J(t), L(J(t)))-s \eta d_{b}(J(t), L(J(t))) \leq & \varphi d_{b}(J(t), L(J(t)))+\omega d_{b}(t, J(t)) \\
& +s \eta d_{b}(t, J(t))+\lambda d_{b}(t, J(t)) .
\end{aligned}
$$

Thus

$$
d_{b}(J(t), L(J(t))) \leq \frac{\omega+s \eta+\lambda}{1-s \eta-\varphi} d_{b}(t, J(t))
$$

\section{Main Results}

In this section, we consider the problem of the existence and uniqueness of common fixed points of two mappings on a complete $b$-metric space.

\subsection{Existence and Uniqueness of Common Fixed Points for Two Mappings}

Theorem 3. Let $L, J: V \rightarrow V$ be two mappings on a complete b-metric space $\left(V, d_{b}\right)$ with $s \geq 1$. Suppose that $F:[0,+\infty) \rightarrow \mathcal{R}$ is a non-decreasing function, and there exist $\tau>0,0<\varphi, \omega<1$ and $0 \leq \mu, \eta, \lambda<1$ satisfying the following properties:

(1) $\varphi+\omega+2 s \mu+\lambda<1$ and $\varphi+\omega+2 s \eta+\lambda<1$,

(2) $s^{2}<\frac{1}{p q}$ and $\frac{s \varphi}{1-\eta}<1$, and

(3) for $u, t \in V$, the inequality $d_{b}(L(u), J(t))>0$ implies

$\tau+F\left(d_{b}(L(u), J(t))\right) \leq F\left(\varphi d_{b}(u, L(u))+\omega d_{b}(t, J(t))+\mu d_{b}(u, J(t))+\eta d_{b}(t, L(u))+\lambda d_{b}(u, t)\right)$.

Then $L$ and $J$ have a unique common fixed point.

Proof. Let $u$ be an element of $V$. Put $u_{0}=u$. For each $n \in \mathcal{N}$, we define

$$
u_{2 n-1}=L\left(u_{2 n-2}\right) \text { and } u_{2 n}=J\left(u_{2 n-1}\right) .
$$

We consider the following four cases:

Case I. If $u_{0}=u_{1}$, that is $u_{0}=L\left(u_{0}\right)$, then $u_{0}=J\left(u_{0}\right)$. Indeed, if $u_{0} \neq J\left(u_{0}\right)=$ $J\left(L\left(u_{0}\right)\right)=J\left(u_{1}\right)$, then $d_{b}\left(L\left(u_{0}\right), J\left(u_{1}\right)\right)>0$. From condition (3), it follows that

$$
\begin{aligned}
F\left(d_{b}\left(L\left(u_{0}\right), J\left(u_{1}\right)\right)\right)< & \tau+F\left(d_{b}\left(L\left(u_{0}\right), J\left(u_{1}\right)\right)\right. \\
\leq & F\left(\varphi d_{b}\left(u_{0}, L\left(u_{0}\right)\right)+\omega d_{b}\left(u_{1}, J\left(u_{1}\right)\right)+\mu d_{b}\left(u_{0}, J\left(u_{1}\right)\right)\right. \\
& \left.+\eta d_{b}\left(u_{1}, L\left(u_{0}\right)\right)+\lambda d_{b}\left(u_{0}, u_{1}\right)\right) \\
= & F\left(\omega d_{b}\left(u_{1}, J\left(u_{1}\right)\right)+\mu d_{b}\left(u_{0}, J\left(u_{1}\right)\right)\right) .
\end{aligned}
$$

Since $F$ is non-decreasing, we obtain

$$
(1-\omega-s \mu) d_{b}\left(L\left(u_{0}\right), J\left(u_{1}\right)\right) \leq 0,
$$

which yields $1-\omega-s \mu \leq 0$, a contradiction. Hence, $u_{0}$ is a common fixed point of the mappings $L$ and $J$.

Case II. If $u_{1}=u_{2}$, that is $u_{1}=J\left(u_{1}\right)$, then $u_{1}=L\left(u_{1}\right)$. If $u_{1} \neq L\left(u_{1}\right)=L\left(J\left(u_{1}\right)\right)=$ $L\left(u_{2}\right)$, then $d_{b}\left(J\left(u_{1}\right), L\left(u_{2}\right)\right)>0$. From condition (3) and the fact that $F$ is non-decreasing, we have

$$
\begin{aligned}
d_{b}\left(L\left(u_{2}\right), J\left(u_{1}\right)\right) \leq & \varphi d_{b}\left(u_{2}, L\left(u_{2}\right)+\omega d_{b}\left(u_{1}, J\left(u_{1}\right)\right)+\mu d_{b}\left(u_{2}, J\left(u_{1}\right)\right)\right. \\
& \left.+\eta d_{b}\left(u_{1}, L\left(u_{2}\right)\right)+\lambda d_{b}\left(u_{2}, u_{1}\right)\right) .
\end{aligned}
$$

By Lemma 1, we get

$$
(1-\varphi-s \eta) d_{b}\left(L\left(u_{2}\right), J\left(u_{1}\right)\right) \leq 0,
$$


which implies $d_{b}\left(L\left(u_{2}\right), J\left(u_{1}\right)\right)=0$, a contradiction. Hence, $u_{1}$ is a common fixed point of $L$ and $J$.

Case III. Similarly, if $u_{n}=u_{n+1}$ for some $n$, we can also obtain that $u_{n}$ is a common fixed point of $L$ and $J$.

Case IV. If $u_{0} \neq u_{1}$ and $u_{1} \neq u_{2}$, then from condition (1), we get

$$
\varphi+\omega+s \mu+s \eta+\lambda<1
$$

Combining with Lemma 1, we deduce that

$$
\begin{aligned}
& d_{b}\left(u_{1}, u_{2}\right) \leq p d_{b}\left(u_{0}, f\left(u_{0}\right)\right), \\
& d_{b}\left(u_{2}, u_{3}\right) \leq q d_{b}\left(u_{1}, u_{2}\right) \leq p q d_{b}\left(u_{0}, f\left(u_{0}\right)\right),
\end{aligned}
$$

where $p=\frac{\varphi+s \mu+\lambda}{1-s \mu-\omega}, q=\frac{\omega+s \eta+\lambda}{1-s \eta-\varphi}$ and $0 \leq p, q<1$. Repeating this process, it is not difficult to see that

$$
d_{b}\left(u_{n}, u_{n+1}\right) \leq \begin{cases}p^{\frac{n}{2}} q^{\frac{n}{2}} d_{b}\left(u_{0}, f\left(u_{0}\right)\right), & \text { if } n \text { is even, } \\ p^{\frac{n+1}{2}} q^{\frac{n-1}{2}} d_{b}\left(u_{0}, f\left(u_{0}\right)\right), & \text { if } n \text { is odd. }\end{cases}
$$

Now, we will verify that $\left\{u_{n}\right\}_{n \in N}$ is a Cauchy sequence. To this end, we discuss the following two cases.

$\left(b_{1}\right)$ Let $m=n+i$, if $i$ is odd and $i>2$, we have

$$
\begin{aligned}
d_{b}\left(u_{n}, u_{m}\right) \leq & s\left(d_{b}\left(u_{n}, u_{n+1}\right)+d_{b}\left(u_{n+1}, u_{m}\right)\right) \\
\leq & s d_{b}\left(u_{n}, u_{n+1}\right)+s^{2} d_{b}\left(u_{n+1}, u_{n+2}\right)+s^{2} d_{b}\left(u_{n+2}, u_{m}\right) \\
\leq & s d_{b}\left(u_{n}, u_{n+1}\right)+s^{2} d_{b}\left(u_{n+1}, u_{n+2}\right)+s^{3} d_{b}\left(u_{n+2}, u_{n+3}\right)+s^{3} d_{b}\left(u_{n+3}, u_{m}\right) \\
\leq & s d_{b}\left(u_{n}, u_{n+1}\right)+s^{2} d_{b}\left(u_{n+1}, u_{n+2}\right)+s^{3} d_{b}\left(u_{n+2}, u_{n+3}\right) \\
& +s^{4} d_{b}\left(u_{n+3}, u_{n+4}\right)+\cdots+s^{m-n-1} d_{b}\left(u_{m-2}, u_{m-1}\right)+s^{m-n-1} d_{b}\left(u_{m-1}, u_{m}\right) .
\end{aligned}
$$

In this case, when $n$ is even, we obtain

$$
\begin{aligned}
& d_{b}\left(u_{n}, u_{m}\right) \leq s p^{\frac{n}{2}} q^{\frac{n}{2}} d_{b}\left(u_{0}, u_{1}\right)+s^{2} p^{\frac{n+2}{2}} q^{\frac{n}{2}} d_{b}\left(u_{0}, u_{1}\right) \\
& +s^{3} p^{\frac{n+2}{2}} q^{\frac{n+2}{2}} d_{b}\left(u_{0}, u_{1}\right)+s^{4} p^{\frac{n+4}{2}} q^{\frac{n+2}{2}} d_{b}\left(u_{0}, u_{1}\right)+\ldots \\
& +s^{m-n-1}\left(p^{\frac{m-1}{2}} q^{\frac{m-3}{2}}+p^{\frac{m-1}{2}} q^{\frac{m-1}{2}}\right) d_{b}\left(u_{0}, u_{1}\right) \\
& =s p^{\frac{n}{2}} q^{\frac{n}{2}}\left(1+s p+s^{2} p q+s^{3} p^{2} q+s^{4} p^{2} q^{2}+\ldots\right. \\
& \left.+s^{m-n-2} p^{\frac{m-n-1}{2}} q^{\frac{m-n-3}{2}}+s^{m-n-2} p^{\frac{m-n-1}{2}} q^{\frac{m-n-1}{2}}\right) d_{b}\left(u_{0}, u_{1}\right) \\
& =s p^{\frac{n}{2}} q^{\frac{n}{2}}\left[\left(1+s^{2} p q+s^{4} p^{2} q^{2}+\cdots+s^{m-n-1} p^{\frac{m-n-1}{2}} q^{\frac{m-n-1}{2}}\right)\right. \\
& \left.+\left(s p+s^{3} p^{2} q+s^{5} p^{3} q^{2}+\cdots+s^{m-n-2} p^{\frac{m-n-1}{2}} q^{\frac{m-n-3}{2}}\right)\right] d_{b}\left(u_{0}, u_{1}\right) \\
& =\left[\frac{1-s^{m-n+1} p^{\frac{m-n+1}{2}} q^{\frac{m-n+1}{2}}}{1-s^{2} p q}+\frac{s p\left(1-s^{m-n-1} p^{\frac{m-n-1}{2}} q^{\frac{m-n-1}{2}}\right)}{1-s^{2} p q}\right] \\
& s p^{\frac{n}{2}} q^{\frac{n}{2}} d_{b}\left(u_{0}, u_{1}\right) \\
& =\left[\frac{1-s^{i+1} p^{\frac{i+1}{2}} q^{\frac{i+1}{2}}}{1-s^{2} p q}+\frac{s p\left(1-s^{i-1} p^{\frac{i-1}{2}} q^{\frac{i-1}{2}}\right)}{1-s^{2} p q}\right] s p^{\frac{n}{2}} q^{\frac{n}{2}} d_{b}\left(u_{0}, u_{1}\right) \\
& =\left[\frac{1+s p-(1+s q) s^{i} p^{\frac{i+1}{2}} q^{\frac{i-1}{2}}}{1-s^{2} p q}\right] s p^{\frac{n}{2}} q^{\frac{n}{2}} d_{b}\left(u_{0}, u_{1}\right) .
\end{aligned}
$$


When $n$ is odd, we get

$$
\begin{aligned}
d_{b}\left(u_{n}, u_{m}\right) \leq & s p^{\frac{n+1}{2}} q^{\frac{n-1}{2}} d_{b}\left(u_{0}, u_{1}\right)+s^{2} p^{\frac{n+1}{2}} q^{\frac{n+1}{2}} d_{b}\left(u_{0}, u_{1}\right) \\
& +s^{3} p^{\frac{n+3}{2}} q^{\frac{n+1}{2}} d_{b}\left(u_{0}, u_{1}\right)+s^{4} p^{\frac{n+3}{2}} q^{\frac{n+3}{2}} d_{b}\left(u_{0}, u_{1}\right)+\ldots \\
& +s^{m-n-1}\left(p^{\frac{m-2}{2}} q^{\frac{m-2}{2}}+p^{\frac{m}{2}} q^{\frac{m-2}{2}}\right) d_{b}\left(u_{0}, u_{1}\right) \\
= & s p^{\frac{n+1}{2}} q^{\frac{n-1}{2}}\left(1+s q+s^{2} p q+s^{3} p q^{2}+s^{4} p^{2} q^{2}+\ldots\right. \\
& \left.+s^{m-n-2} p^{\frac{m-n-3}{2}} q^{\frac{m-n-1}{2}}+s^{m-n-2} p^{\frac{m-n-1}{2}} q^{\frac{m-n-1}{2}}\right) d_{b}\left(u_{0}, u_{1}\right) \\
= & s p^{\frac{n+1}{2}} q^{\frac{n-1}{2}}\left[\left(1+s^{2} p q+s^{4} p^{2} q^{2}+\cdots+s^{m-n-1} p^{\frac{m-n-1}{2}} q^{\frac{m-n-1}{2}}\right)\right. \\
& \left.+\left(s q+s^{3} p q^{2}+s^{5} p^{2} q^{3}+\cdots+s^{m-n-2} p^{\frac{m-n-3}{2}} q^{\frac{m-n-1}{2}}\right)\right] d_{b}\left(u_{0}, u_{1}\right) \\
= & {\left[\frac{1-s^{m-n+1} p^{\frac{m-n+1}{2}} q^{\frac{m-n+1}{2}}}{1-s^{2} p q}+\frac{s q\left(1-s^{m-n-1} p^{\frac{m-n-1}{2}} q^{\frac{m-n-1}{2}}\right)}{1-s^{2} p q}\right] } \\
& s p^{\frac{n+1}{2}} q^{\frac{n-1}{2}} d_{b}\left(u_{0}, u_{1}\right) \\
= & {\left[\frac{1-s^{i+1} p^{\frac{i+1}{2}} q^{\frac{i+1}{2}}}{1-s^{2} p q}+\frac{s q\left(1-s^{i-1} p^{\frac{i-1}{2}} q^{\frac{i-1}{2}}\right)}{1-s^{2} p q}\right] s p^{\frac{n+1}{2}} q^{\frac{n-1}{2}} d_{b}\left(u_{0}, u_{1}\right) } \\
= & {\left[\frac{\left.1+s q-(1+s p) s^{i} p^{\frac{i-1}{2}} q^{\frac{i+1}{2}}\right] s p^{\frac{n+1}{2}} q^{\frac{n-1}{2}} d_{b}\left(u_{0}, u_{1}\right) .}{1-s^{2} p q}\right.}
\end{aligned}
$$

$\left(b_{2}\right)$ If $i$ is even and $i>2$, by the similar argument, we deduce that

$$
\begin{aligned}
d_{b}\left(u_{n}, u_{m}\right) \leq & s p^{\frac{n}{2}} q^{\frac{n}{2}} d_{b}\left(u_{0}, u_{1}\right)+s^{2} p^{\frac{n+2}{2}} q^{\frac{n}{2}} d_{b}\left(u_{0}, u_{1}\right) \\
& +s^{3} p^{\frac{n+2}{2}} q^{\frac{n+2}{2}} d_{b}\left(u_{0}, u_{1}\right)+s^{4} p^{\frac{n+4}{2}} q^{\frac{n+2}{2}} d_{b}\left(u_{0}, u_{1}\right)+\ldots \\
& +s^{m-n-1}\left(p^{\frac{m-2}{2}} q^{\frac{m-2}{2}}+p^{\frac{m}{2}} q^{\frac{m-2}{2}}\right) d_{b}\left(u_{0}, u_{1}\right) \\
\leq & s p^{\frac{n}{2}} q^{\frac{n}{2}}\left(1+s p+s^{2} p q+s^{3} p^{2} q+s^{4} p^{2} q^{2}+\ldots\right. \\
& \left.+s^{m-n-2} p^{\frac{m-n-2}{2}} q^{\frac{m-n-2}{2}}+s^{m-n-2} p^{\frac{m-n}{2}} q^{\frac{m-n-2}{2}}\right) d_{b}\left(u_{0}, u_{1}\right) \\
\leq & s p^{\frac{n}{2}} q^{\frac{n}{2}}\left[\left(1+s^{2} p q+s^{4} p^{2} q^{2}+\cdots+s^{m-n-2} p^{\frac{m-n-2}{2}} q^{\frac{m-n-2}{2}}\right)\right. \\
& \left.+\left(s p+s^{3} p^{2} q+s^{5} p^{3} q^{2}+\cdots+s^{m-n-2} p^{\frac{m-n}{2}} q^{\frac{m-n-2}{2}}\right)\right] d_{b}\left(u_{0}, u_{1}\right) \\
\leq & s p^{\frac{n}{2}} q^{\frac{n}{2}}(1+s p) \frac{1-s^{m-n} p^{\frac{m-n}{2}} q^{\frac{m-n}{2}}}{1-s^{2} p q} d_{b}\left(u_{0}, u_{1}\right) \\
\leq & s p^{\frac{n}{2}} q^{\frac{n}{2}}(1+s p) \frac{1-s^{i} p^{\frac{i}{2}} q^{\frac{i}{2}}}{1-s^{2} p q} d_{b}\left(u_{0}, u_{1}\right),
\end{aligned}
$$

where $n$ and $m$ are even. Moreover,

$$
\begin{aligned}
d_{b}\left(u_{n}, u_{m}\right) \leq & s p^{\frac{n+1}{2}} q^{\frac{n-1}{2}} d_{b}\left(u_{0}, u_{1}\right)+s^{2} p^{\frac{n+1}{2}} q^{\frac{n+1}{2}} d_{b}\left(u_{0}, u_{1}\right) \\
& +s^{3} p^{\frac{n+3}{2}} q^{\frac{n+1}{2}} d_{b}\left(u_{0}, u_{1}\right)+s^{4} p^{\frac{n+3}{2}} q^{\frac{n+3}{2}} d_{b}\left(u_{0}, u_{1}\right)+\ldots \\
& +s^{m-n-1}\left(p^{\frac{m-1}{2}} q^{\frac{m-3}{2}}+p^{\frac{m-1}{2}} q^{\frac{m-1}{2}}\right) d_{b}\left(u_{0}, u_{1}\right) \\
\leq & s p^{\frac{n+1}{2}} q^{\frac{n-1}{2}}(1+s q) \frac{1-s^{m-n} p^{\frac{m-n}{2}} q^{\frac{m-n}{2}}}{1-s^{2} p q} d_{b}\left(u_{0}, u_{1}\right) \\
\leq & s p^{\frac{n+1}{2}} q^{\frac{n-1}{2}}(1+s q) \frac{1-s^{i} p^{\frac{i}{2}} q^{\frac{i}{2}}}{1-s^{2} p q} d_{b}\left(u_{0}, u_{1}\right)
\end{aligned}
$$


where $n$ is odd and $m$ is even. Thus,

$$
\begin{aligned}
& d_{b}\left(u_{n}, u_{m}\right) \leq \\
& \begin{cases}s p^{\frac{n}{2}} q^{\frac{n}{2}}\left[\frac{1+s p-(1+s q) s^{i} p^{\frac{i+1}{2}} q^{\frac{i-1}{2}}}{1-s^{2} p q}\right] d_{b}\left(u_{0}, u_{1}\right), & \text { if } i \text { is odd and } n \text { is even, } \\
s p^{\frac{n+1}{2}} q^{\frac{n-1}{2}}\left[\frac{1+s q-(1+s p) s^{i} p^{\frac{i-1}{2}} q^{\frac{i+1}{2}}}{1-s^{2} p q}\right] d_{b}\left(u_{0}, u_{1}\right), & \text { if } i \text { is odd and } n \text { is odd, } \\
s p^{\frac{n}{2}} q^{\frac{n}{2}}(1+s p) \frac{1-s^{i} p^{\frac{i}{2}} q^{\frac{i}{2}}}{1-s^{2} p q} d_{b}\left(u_{0}, u_{1}\right), & \text { if } i \text { is even and } n \text { is even, } \\
s p^{\frac{n+1}{2}} q^{\frac{n-1}{2}}(1+s q) \frac{1-s^{i} p^{\frac{i}{2}} q^{\frac{i}{2}}}{1-s^{2} p q} d_{b}\left(u_{0}, u_{1}\right), & \text { if } i \text { is even and } n \text { is odd. }\end{cases}
\end{aligned}
$$

Letting $n, m \rightarrow \infty$, we obtain that $d_{b}\left(u_{n}, u_{m}\right) \rightarrow 0$, since $0 \leq p, q<1$. Hence, we draw the conclusion that $\left\{u_{n}\right\}$ is a Cauchy sequence. Noticing that the completeness of the space, there exists $r \in V$ such that

$$
\lim _{n \rightarrow \infty} d_{b}\left(u_{n}, r\right)=0 \text {. }
$$

Next, we verify that $r$ is a common fixed point of $L$ and $J$. Indeed, if $d_{b}(L(r), r)>0$, condition (3) yields

$$
\begin{aligned}
F\left(d_{b}\left(L(r), u_{2 n}\right)\right)< & \tau+F\left(d_{b}\left(L(r), u_{2 n}\right)\right)=\tau+F\left(d_{b}\left(L(r), J\left(u_{2 n-1}\right)\right)\right) \\
\leq & F\left(\varphi d_{b}(r, L(r))+\omega d_{b}\left(u_{2 n-1}, u_{2 n}\right)+\mu d_{b}\left(r, u_{2 n}\right)\right. \\
& \left.+\eta d_{b}\left(u_{2 n-1}, L(r)\right)+\lambda d_{b}\left(r, u_{2 n-1}\right)\right) .
\end{aligned}
$$

Since $F$ is non-decreasing, we get

$$
\begin{aligned}
d_{b}\left(L(r), u_{2 n}\right) \leq & \varphi d_{b}(r, L(r))+\omega d_{b}\left(u_{2 n-1}, u_{2 n}\right)+\mu d_{b}\left(r, u_{2 n}\right) \\
& +\eta d_{b}\left(u_{2 n-1}, L(r)\right)+\lambda d_{b}\left(r, u_{2 n-1}\right) .
\end{aligned}
$$

Moreover, from condition $\left(a_{3}\right)$ of Definition 1, it follows that

$$
d_{b}(L(r), r) \leq s\left[d_{b}\left(L(r), u_{2 n}\right)+d_{b}\left(u_{2 n}, r\right)\right]
$$

Then

$$
\frac{1}{s} d_{b}(L(r), r) \leq \lim _{n \rightarrow \infty} \sup d_{b}\left(L(r), u_{2 n}\right) \leq \frac{\varphi}{1-s \eta} d_{b}(L(r), r) .
$$

Hence, we deduce that $\frac{1}{s} \leq \frac{\varphi}{1-s \eta}$ which contradicts the fact $\frac{s \varphi}{1-s \eta}<1$. Therefore, we obtain $d_{b}(L(r), r)=0$. Similarly, we can get $J(r)=r$. Therefore, we can write

$$
L(r)=J(r)=r .
$$

For the uniqueness, we assume that $r$ and $r^{*}$ are two distinct common fixed points of $f$ and $g$. Then

$$
\begin{aligned}
\tau+F\left(d_{b}\left(r, r^{*}\right)\right)= & \tau+F\left(d_{b}\left(L(r), J\left(r^{*}\right)\right)\right) \\
\leq & F\left(\varphi d_{b}(r, L(r))+\omega d_{b}\left(r^{*}, J\left(r^{*}\right)\right)+\mu d_{b}\left(r, J\left(r^{*}\right)\right)\right. \\
& \left.+\eta d_{b}\left(r^{*}, L(r)\right)+\lambda d_{b}\left(r, r^{*}\right)\right) \\
= & F\left(\mu d_{b}\left(r, J\left(r^{*}\right)\right)+\eta d_{b}\left(r^{*}, L(r)\right)+\lambda d_{b}\left(r, r^{*}\right)\right) .
\end{aligned}
$$

Since $F$ is non-decreasing, we deduce that

$$
d_{b}\left(r, r^{*}\right) \leq \mu d_{b}\left(r, J\left(r^{*}\right)\right)+\eta d_{b}\left(r^{*}, L(r)\right)+\lambda d_{b}\left(r, r^{*}\right),
$$

which implies

$$
(1-\lambda-\mu-\eta) d_{b}\left(r, r^{*}\right) \leq 0
$$


Then

$$
1-\lambda-\mu-\eta \leq 0,
$$

which is a contradiction. Hence, $r=r^{*}$.

The following example shows the validity of Theorem 3.

Example 3. Let $V=[0,8]$, and $L, J: V \rightarrow V$ be two mappings defined by

$$
L(u)= \begin{cases}7, & \text { if } u \in(0,8] \\ 8, & \text { if } u=0\end{cases}
$$

and

$$
J(u)= \begin{cases}7, & \text { if } u \in(0,8] \\ 6, & \text { if } u=0 .\end{cases}
$$

We define a $b$-metric $d_{b}: V \times V \rightarrow[0, \infty)$ by

$$
d_{b}(u, t)=(u-t)^{2}, \quad \text { for all } u, t \in V
$$

Clearly, $\left(V, d_{b}\right)$ is a complete b-metric space with constant $s=2$ (see [25] for details). We observe that $d_{b}(L(u), J(t))>0$, when $(u, t) \in D=\{(u, t): u \in(0,8], t=0\} \cup\{(u, t): u=$ $0, t \in(0,8]\} \cup\{(u, t): u=0, t=0\}$.

Define $E: V \times V \rightarrow[0, \infty)$ by

$$
E(u, t)=\frac{1}{4} d_{b}(u, L(u))+\frac{1}{4} d_{b}(t, J(u))+\frac{1}{16} d_{b}(u, t), u, t \in V .
$$

Next, we discuss the following three cases:

(i) If $u \in(0,8]$ and $t=0$, then we obtain

$$
\begin{aligned}
\frac{1}{6}-\frac{1}{d_{b}(L(u), J(0))+1} & \leq \frac{1}{6}-\frac{1}{2}=-\frac{1}{3} \\
& <-\frac{4}{36}=-\frac{4}{d_{b}(0, J(0))} \\
& \leq-\frac{1}{E(u, 0)} \\
& <-\frac{1}{E(u, 0)+1}
\end{aligned}
$$

(ii) If $t \in(0,8]$ and $u=0$, then we have

$$
\begin{aligned}
\frac{1}{6}-\frac{1}{d_{b}(L(0), J(t))+1} & \leq \frac{1}{6}-\frac{1}{2}=-\frac{1}{3} \\
& <-\frac{4}{64}=-\frac{4}{d_{b}(0, f(0))} \\
& \leq-\frac{1}{E(0, t)} \\
& <-\frac{1}{E(0, t)+1}
\end{aligned}
$$


(iii) If $t=0$ and $u=0$, then we get

$$
\begin{aligned}
\frac{1}{6}-\frac{1}{d_{b}(L(0), J(0))+1} & \leq \frac{1}{6}-\frac{1}{3} \\
& <-\frac{1}{25} \\
& \leq-\frac{1}{E(0,0)} \\
& <-\frac{1}{E(0,0)+1} .
\end{aligned}
$$

Combining with (3), (4), and (5), we deduce that

$$
\frac{1}{6}-\frac{1}{d_{b}(L(u), J(t))+1}<-\frac{1}{E(u, t)+1},
$$

for all $(u, t) \in D$.

Therefore, if we consider $F(h)=-\frac{1}{h+1}$, where $h \geq 0, t \in(0, \infty)$, and $\tau=\frac{1}{6}$, then all the conditions of Theorem 3 are satisfied with $\varphi=\frac{1}{4}, \omega=\frac{1}{4}, \mu=\eta=0, \lambda=\frac{1}{16}$. Meanwhile, we notice that $L(z)=J(z)=z$ if and only if $z=7$. Hence, $L$ and $J$ have a unique common fixed point.

Next, we give the following result which shows the uniqueness and existence of the common fixed point for two mappings in a complete $b$-metric space by virtue of the function $\theta$.

Theorem 4. Let $L$ and $J$ be two self-maps on a complete b-metric space $\left(V, d_{b}\right)$ with constant $s \geq 1$. Let $\varphi, \omega \in(0,1), \mu, \eta, \lambda \in[0,1)$ be the constants with $\varphi+\omega+s \mu+s \eta+\lambda<1$, $s^{2} \varphi+s^{2} \eta+\left(s^{3}+s^{4}\right)(\mu+\lambda)<1$, and $s^{2} \omega+s^{2} \mu+\left(s^{3}+s^{4}\right)(\eta+\lambda)<1$. Define $\theta:[0,1] \times$ $[0,1] \times[0,1] \times[0,1] \times[0,1] \rightarrow(0,1]$ by

$$
\begin{aligned}
\theta(\varphi, \omega, \mu, \eta, \lambda)= & \min \left\{\frac{1-s^{2} \varphi-s^{2} \eta-\left(s^{3}+s^{4}\right)(\mu+\lambda)}{1-s^{3} \mu-s^{2} \eta-s^{3} \lambda},\right. \\
& \left.\frac{1-s^{2} \omega-s^{2} \mu-\left(s^{3}+s^{4}\right)(\eta+\lambda)}{1-s^{2} \mu-s^{3} \eta-s^{3} \lambda}\right\} .
\end{aligned}
$$

Suppose that each of the conditions

$$
\begin{aligned}
& \theta(\varphi, \omega, \mu, \eta, \lambda) d_{b}(u, L(u)) \leq s^{2} d_{b}(u, t) \quad \text { or } \\
& \theta(\varphi, \omega, \mu, \eta, \lambda) d_{b}(t, J(t)) \leq s^{2} d_{b}(u, t),
\end{aligned}
$$

implies

$$
\begin{aligned}
d_{b}(L(u), J(t)) \leq & \varphi d_{b}(u, L(u))+\omega d_{b}(t, J(t))+\mu d_{b}(u, J(t))+\eta d_{b}(t, L(u)) \\
& +\lambda d_{b}(u, t),
\end{aligned}
$$

for $u, t \in V$. Then $L$ and $J$ have a unique common fixed point $r$.

Proof. It is not difficult to see that $\theta(\varphi, \omega, \mu, \eta, \lambda) \in(0,1)$, since

$$
1-s^{2} \varphi-s^{2} \eta-\left(s^{3}+s^{4}\right)(\mu+\lambda)<1-s^{3} \mu-s^{2} \eta-s^{3} \lambda,
$$

and

$$
1-s^{2} \omega-s^{2} \mu-\left(s^{3}+s^{4}\right)(\eta+\lambda)<1-s^{2} \mu-s^{3} \eta-s^{3} \lambda
$$


Let $e \in V$. Put $e_{0}=e$. For each $n \in \mathcal{N}$, we define $e_{2 n+1}=L\left(e_{2 n}\right)$ and $e_{2 n}=J\left(e_{2 n-1}\right)$. If $e_{0}=e_{1}$, that is $e_{0}=L\left(e_{0}\right)$, then $e_{0}=J\left(e_{0}\right)$. Indeed, if $e_{0} \neq J\left(e_{0}\right)=J\left(L\left(e_{0}\right)\right)=J\left(e_{1}\right)$, then

$$
\theta(\varphi, \omega, \mu, \eta, \lambda) d_{b}\left(e_{0}, L\left(e_{0}\right)\right) \leq s^{2} d_{b}\left(e_{0}, L\left(e_{0}\right)\right) .
$$

Hence, we have

$$
\begin{aligned}
d_{b}\left(e_{1}, e_{2}\right) & =d_{b}\left(L\left(e_{0}\right), J\left(e_{1}\right)\right) \\
& \leq \varphi d_{b}\left(e_{0}, e_{1}\right)+\omega d_{b}\left(e_{1}, e_{2}\right)+s \mu\left[d_{b}\left(e_{0}, e_{1}\right)+d_{b}\left(e_{1}, e_{2}\right)\right]+\lambda d_{b}\left(e_{0}, e_{1}\right)
\end{aligned}
$$

It yields

$$
(1-\omega-s \mu) d_{b}\left(e_{1}, e_{2}\right) \leq 0,
$$

which shows that $d_{b}\left(e_{0}, J\left(e_{1}\right)\right)=0$, a contradiction. Hence, $e_{0}$ is a common fixed point of the mappings $L$ and $J$.

Similarly, as in Case II and Case III of Theorem 3, we conclude that if $e_{n}=e_{n+1}$ for some $n$, then $e_{n}$ is also the common fixed point of the mappings $L$ and $J$.

If $e_{0} \neq e_{1}$ and $e_{1} \neq e_{2}$, we notice that

$$
\theta(\varphi, \omega, \mu, \eta, \lambda) d_{b}\left(e_{0}, L\left(e_{0}\right)\right) \leq s^{2} d_{b}\left(e_{0}, L\left(e_{0}\right)\right),
$$

since $\theta(\varphi, \omega, \mu, \eta, \lambda)<1 \leq s^{2}$. Then, we have

$$
\begin{aligned}
d_{b}\left(e_{1}, e_{2}\right) & =d_{b}\left(L\left(e_{0}\right), J\left(e_{1}\right)\right) \\
& \leq \varphi d_{b}\left(e_{0}, e_{1}\right)+\omega d_{b}\left(e_{1}, e_{2}\right)+\mu d_{b}\left(e_{0}, e_{2}\right)+\lambda d_{b}\left(e_{0}, e_{1}\right) .
\end{aligned}
$$

From Lemma 1, it follows that

$$
d_{b}\left(e_{1}, e_{2}\right) \leq p d_{b}\left(e_{0}, e_{1}\right), \text { where } p=\frac{\varphi+s \mu+\lambda}{1-s \mu-\omega} \in(0,1) .
$$

Similarly, we can also deduce that

$$
\theta(\varphi, \omega, \mu, \eta, \lambda) d_{b}\left(e_{1}, e_{2}\right) \leq s^{2} d_{b}\left(e_{1}, e_{2}\right)
$$

From Lemma 1, we obtain

$$
d_{b}\left(e_{2}, e_{3}\right) \leq q d_{b}\left(d_{b}\left(e_{1}, e_{2}\right)\right) \leq p q d_{b}\left(e_{0}, e_{1}\right), \quad \text { where } \quad q=\frac{\omega+s \eta+\lambda}{1-s \eta-\varphi} \in(0,1) .
$$

By repeating the above process, we can establish using a similar argument as in Theorem 3 that $\left\{e_{n}\right\}$ is a Cauchy sequence and $\lim _{n \rightarrow \infty} e_{n}=r$ for some $r \in V$. Now we will prove that $r$ is a common fixed point of the mappings $L$ and $J$. Let $u \neq r$ be an arbitrary element of $V$. Then there exists $N \in \mathcal{N}$ such that $d_{b}\left(e_{n}, r\right) \leq \frac{1}{2+s} d_{b}(u, r)$ for all $n \geq \mathcal{N}$. Hence, we have

$$
d_{b}(u, r) \leq s\left[d_{b}\left(u, e_{2 n-1}\right)+d_{b}\left(e_{2 n-1}, r\right)\right] \leq s\left[d_{b}\left(u, e_{2 n-1}\right)+\frac{1}{2+s} d_{b}(u, r)\right],
$$

for large enough $n$. Thus

$$
\frac{2}{2+s} d_{b}(u, r) \leq s d_{b}\left(u, e_{2 n-1}\right) .
$$


What is more,

$$
\begin{aligned}
\theta(\varphi, \omega, \mu, \eta, \lambda) d_{b}\left(e_{2 n-1}, J\left(e_{2 n-1}\right)\right) & \leq d_{b}\left(e_{2 n-1}, J\left(e_{2 n-1}\right)\right) \\
& \leq s\left[\left(d_{b}\left(e_{2 n-1}, r\right)+d_{b}\left(r, e_{2 n}\right)\right)\right] \\
& \leq \frac{2 s}{2+s} d_{b}(u, r) \\
& \leq s^{2} d_{b}\left(u, e_{2 n-1}\right) .
\end{aligned}
$$

Hence, we deduce that

$$
\begin{aligned}
d_{b}\left(L(u), J\left(e_{2 n-1}\right)\right) \leq & \varphi d_{b}(u, L(u))+\omega d_{b}\left(e_{2 n-1}, J\left(e_{2 n-1}\right)\right) \\
& +\mu d_{b}\left(u, J\left(e_{2 n-1}\right)\right)+\eta d_{b}\left(e_{2 n-1}, L(u)\right)+\lambda d_{b}\left(u, e_{2 n-1}\right) .
\end{aligned}
$$

Then

$$
\begin{aligned}
d_{b}(L(u), r) \leq & s\left[d_{b}\left(L(u), e_{2 n}\right)+d_{b}\left(e_{2 n}, r\right)\right] \\
\leq & s \varphi d_{b}(u, L(u))+s \omega d_{b}\left(e_{2 n-1}, J\left(e_{2 n-1}\right)\right) \\
& +s^{2} \mu\left[d_{b}(u, r)+d_{b}\left(r, e_{2 n}\right)\right]+s^{2} \eta\left[d_{b}\left(e_{2 n-1}, r\right)+d_{b}(L(u), r)\right] \\
& +s^{2} \lambda\left[d_{b}(u, r)+d_{b}\left(r, e_{2 n-1}\right)\right]+s d_{b}\left(e_{2 n}, r\right) \\
\leq & \lim _{n \rightarrow \infty} \sup \left\{s \varphi d_{b}(u, L(u))+s \omega d_{b}\left(e_{2 n-1}, J\left(e_{2 n-1}\right)\right)\right. \\
& +s^{2} \mu\left[d_{b}(u, r)+d_{b}\left(r, e_{2 n}\right)\right]+s^{2} \eta\left[d_{b}\left(e_{2 n-1}, r\right)+d_{b}(L(u), r)\right] \\
& \left.+s^{2} \lambda\left[d_{b}(u, r)+d_{b}\left(r, e_{2 n-1}\right)\right]+s d_{b}\left(e_{2 n}, r\right)\right\} \\
= & s \varphi d_{b}(u, L(u))+s^{2}(\mu+\lambda) d_{b}(u, r)+s^{2} \eta d_{b}(L(u), r) .
\end{aligned}
$$

It follows that

$$
d_{b}(L(r), r) \leq s \varphi d_{b}(L(r), r)+s^{2} \eta d_{b}(L(r), r),
$$

which implies $\left(1-s \varphi-s^{2} \eta\right) d_{b}(L(r), r) \leq 0$. Notice that

$$
1-s \varphi-s^{2} \eta>\left(s^{2}-s\right) \varphi+s^{2} \omega+\left(s^{3}+s^{4}\right)(\mu+\lambda)>0 .
$$

Hence, we deduce $d_{b}(L(r), r)=0$. By adding $-s^{3}(\mu+\lambda) d_{b}(L(u), r)$ on both sides of (6), we have

$$
\begin{aligned}
& d_{b}(L(u), r)-s^{3}(\mu+\lambda) d_{b}(L(u), r) \\
& \leq s \varphi d_{b}(u, L(u))+s^{2}(\mu+\lambda) d_{b}(u, r)-s^{3}(\mu+\lambda) d_{b}(L(u), r)+s^{2} \eta d_{b}(L(u), r) \\
& \leq s \varphi d_{b}(u, L(u))+s^{3}(\mu+\lambda) d_{b}(u, L(u))+s^{2} \eta d_{b}(L(u), r) .
\end{aligned}
$$

Thus

$$
d_{b}(L(u), r) \leq \frac{s \varphi+s^{3} \mu+s^{3} \lambda}{1-s^{3} \mu-s^{2} \eta-s^{3} \lambda} d_{b}(u, L(u)),
$$

and

$$
\begin{aligned}
d_{b}(u, L(u)) & \leq s\left[d_{b}(u, r)+d_{b}(L(u), r)\right] \\
& \leq s d_{b}(u, r)+\frac{s^{2} \varphi+s^{4} \mu+s^{4} \lambda}{1-s^{3} \mu-s^{2} \eta-s^{3} \lambda} d_{b}(u, L(u)) .
\end{aligned}
$$

Therefore, we obtain

$$
\frac{1-s^{2} \varphi-s^{2} \eta-\left(s^{3}+s^{4}\right)(\mu+\lambda)}{1-s^{2} \eta s^{3}-(\mu+\lambda)} d_{b}(u, L(u)) \leq s^{2} d_{b}(u, r) .
$$


Similarly, we can also deduce $d_{b}(J(r), r)=0$ and

$$
\frac{1-s^{2} \omega-s^{2} \mu-\left(s^{3}+s^{4}\right)(\eta+\lambda)}{1-s^{2} \mu-s^{3}(\eta+\lambda)} d_{b}(u, g(u)) \leq s^{2} d_{b}(u, r) .
$$

Hence, $r$ is a common fixed point of the mappings $L$ and $J$. Furthermore, we also have

$$
\theta(\varphi, \omega, \mu, \eta, \lambda) d_{b}(u, L(u)) \leq s^{2} d_{b}(u, r), \theta(\varphi, \omega, \mu, \eta, \lambda) d_{b}(u, J(u)) \leq s^{2} d_{b}(u, r) .
$$

Next, we show the uniqueness of the common fixed point of the mappings $L$ and $J$. Assume that there exist $r, r^{\prime} \in V$ such that $L(r)=J(r)=r$ and $L\left(r^{\prime}\right)=r^{\prime}=J\left(r^{\prime}\right)$. Note that

$$
\theta(\varphi, \omega, \mu, \eta, \lambda) d_{b}(r, L(r)) \leq s^{2} d_{b}\left(r, r^{\prime}\right)
$$

Then we have

$$
\begin{aligned}
d_{b}\left(r, r^{\prime}\right)=d_{b}\left(L(r), J\left(r^{\prime}\right)\right) \leq & \varphi d_{b}(r, L(r))+\omega d_{b}\left(r^{\prime}, J\left(r^{\prime}\right)\right) \\
& +\mu d_{b}\left(r, J\left(r^{\prime}\right)\right)+\eta d_{b}\left(r^{\prime}, L(r)\right)+\lambda d_{b}\left(r, r^{\prime}\right),
\end{aligned}
$$

which yields

$$
(1-\mu-\eta-\lambda) d_{b}\left(r, r^{\prime}\right) \leq 0
$$

Since

$$
1-\mu-\eta-\lambda>\varphi+\omega>0,
$$

we get $d_{b}\left(r, r^{\prime}\right)=0$. This completes the proof.

Example 4. Let $V=[0,1]$ be equipped with b-metric $d_{b}(u, t)=(u-t)^{2}$ and $u, t \in V$, where $s=2$. Define L and $J$ by

$$
L(u)=\frac{u}{2}, \quad J(u)=\frac{u}{3}, \quad u \in V,
$$

and set

$$
E(u, t)=\frac{1}{32} d_{b}(u, L(u))+\frac{1}{32} d_{b}(t, J(t))+\frac{1}{64} d_{b}(u, t)
$$

for all $u, t \in V$. Let $\varphi=\frac{1}{32}, \omega=\frac{1}{32}, \mu=\eta=0, \lambda=\frac{1}{64}$, it is easy to verify that

$$
\begin{aligned}
& \varphi+\omega+s \mu+s \eta+\lambda<1, \\
& s^{2} \varphi+s^{2} \eta+\left(s^{3}+s^{4}\right)(\mu+\lambda)<1, \\
& s^{2} \omega+s^{2} \mu+\left(s^{3}+s^{4}\right)(\eta+\lambda)<1,
\end{aligned}
$$

and

$$
\begin{aligned}
\theta(\varphi, \omega, \mu, \eta, \lambda)= & \min \left\{\frac{1-s^{2} \omega-s^{2} \mu-\left(s^{3}+s^{4}\right) \eta-\left(s^{3}+s^{4}\right) \lambda}{1-s^{2} \mu-s^{3} \eta-s^{3} \lambda}\right. \\
& \left.\frac{1-s^{2} \varphi-\left(s^{3}+s^{4}\right) \mu-s^{2} \eta-\left(s^{3}+s^{4}\right) \lambda}{1-s^{3} \mu-s^{2} \eta-s^{3} \lambda}\right\}=\frac{4}{7}
\end{aligned}
$$

Next, we claim that the condition

$$
\begin{aligned}
& \theta(\varphi, \omega, \mu, \eta, \lambda) d_{b}(u, L(u)) \leq s^{2} d_{b}(u, t) \quad \text { or } \\
& \theta(\varphi, \omega, \mu, \eta, \lambda) d_{b}(t, J(t)) \leq s^{2} d_{b}(u, t),
\end{aligned}
$$


implies

$$
\begin{aligned}
d_{b}(L(u), J(t)) \leq & \varphi d_{b}(u, L(u))+\omega d_{b}(t, J(t))+\mu d_{b}(u, J(t))+\eta d_{b}(t, L(u)) \\
& +\lambda d_{b}(u, t),
\end{aligned}
$$

for $u, t \in V$. Indeed,

$$
d_{b}(L(u), J(t))=\left(\frac{u}{2}-\frac{t}{3}\right)^{2} \leq \frac{1}{128} u^{2}+\frac{1}{72} t^{2}+\frac{1}{64}(u-t)^{2}, u, t \in V .
$$

Moreover, if

$$
\theta(\varphi, \omega, \mu, \eta, \lambda) d_{b}(u, L(u)) \leq s^{2} d_{b}(u, t)
$$

then we deduce

$$
\frac{u^{2}}{28} \leq(u-t)^{2}
$$

Now, we study the following two cases:

(i) If $u>t$, we have

$$
t \leq\left(1-\frac{1}{\sqrt{28}}\right) u
$$

Combining (10) and (12) yields

$$
A u^{2} \leq 0
$$

where

$$
A=\left(\frac{1}{4}-\frac{1}{128}-\frac{1}{64}\right)+\left(\frac{1}{9}-\frac{1}{72}-\frac{1}{64}\right)\left(1-\frac{1}{\sqrt{28}}\right)^{2}+\left(\frac{1}{32}-\frac{1}{3}\right)\left(1-\frac{1}{\sqrt{28}}\right)>0,
$$

which means that $u=t=0$. Therefore, when $u=t=0$, the condition

$$
\theta(\varphi, \omega, \mu, \eta, \lambda) d_{b}(u, L(u)) \leq s^{2} d_{b}(u, t),
$$

implies

$$
\begin{aligned}
d_{b}(L(u), J(t)) \leq & \varphi d_{b}(u, L(u))+\omega d_{b}(t, J(t))+\mu d_{b}(u, J(t))+\eta d_{b}(t, L(u)) \\
& +\lambda d_{b}(u, t) .
\end{aligned}
$$

(ii) If $u<t$, we get

$$
u \leq \frac{\sqrt{28}}{\sqrt{28}+1} t
$$

Then by (10) and (13), we obtain

$$
B t^{2} \leq 0
$$

where

$$
B=\left(\frac{1}{4}-\frac{1}{128}-\frac{1}{64}\right)\left(\frac{\sqrt{28}}{\sqrt{28}+1}\right)^{2}+\left(\frac{1}{9}-\frac{1}{72}-\frac{1}{64}\right)+\left(\frac{1}{32}-\frac{1}{3}\right) \frac{\sqrt{28}}{\sqrt{28}+1}<0 .
$$

Therefore, for $u, t \in[0,1]$, when $u \leq \frac{\sqrt{28}}{\sqrt{28}+1} t$, the condition

$$
\theta(\varphi, \omega, \mu, \eta, \lambda) d_{b}(u, L(u)) \leq s^{2} d_{b}(u, t),
$$


implies

$$
\begin{aligned}
d_{b}(L(u), J(t)) \leq & \varphi d_{b}(u, L(u))+\omega d_{b}(t, J(t))+\mu d_{b}(u, J(t))+\eta d_{b}(t, L(u)) \\
& +\lambda d_{b}(u, t) .
\end{aligned}
$$

As a consequence, we claim that all the conditions of Theorem 4 are satisfied with $\varphi=\frac{1}{32}, \omega=$ $\frac{1}{32}, \mu=\eta=0, \lambda=\frac{1}{64}$. Meanwhile, it is not difficult to see that 0 is the unique common fixed point of the mappings $L$ and $J$.

\subsection{A Generalized Form of Jungck Fixed Point Theorem}

Next, we give a generalized form of Jungck fixed point theorem in complete $b$ metric spaces.

Theorem 5. Let $L$ be a continuous self-map on a complete $b$-metric space $\left(V, d_{b}\right)$. Then $L$ has a fixed point if and only if there exist the constants $\omega \in(0,1), \varphi, \mu, \eta, \lambda \in[0,1)$ with $\varphi+\omega+\mu+$ $2 s \eta+\lambda<1$ and a continuous self-map J on $V$ satisfying the following properties:

(1) $J(V) \subseteq L(V)$,

(2) L and $J$ commute under composition (i.e., $J(L(u))=L(J(u))$ for all $u \in V$ ),

(3) $d_{b}(J(u), J(t)) \leq \varphi d_{b}(L(u), J(u))+\omega d_{b}(L(t), J(t))+\mu d_{b}(L(u), J(t))+\eta d_{b}(L(t)$, $J(u))+\lambda d_{b}(L(u), L(t))$ for all $u, t \in V$.

If the above conditions hold, then $L$ and J have a unique common fixed point.

Proof. If $L$ has a fixed point $r \in V$, then we define $J: V \rightarrow V$ by $J(u)=r$ for all $u \in V$. It follows immediately that $J(V) \subseteq L(V)$. Moreover, for any $u \in V, J(L(u))=r$ and $L(J(u))=L(r)=r$, which implies that $J(L(u))=L(J(u))$ for all $u \in V$. In addition, for any $\varphi, \omega, \mu, \eta, \lambda \in[0,1)$ and $u, t \in V$, we obtain

$$
\begin{aligned}
d_{b}(J(u), J(t))= & d_{b}(r, r)=0 \\
\leq & \varphi d_{b}(L(u), r)+\omega d_{b}(L(t), r)+\mu d_{b}(L(u), r) \\
& +\eta d_{b}(L(t), r)+\lambda d_{b}(L(u), L(t)) .
\end{aligned}
$$

On the other hand, if conditions (1)-(3) are satisfied, we claim that $L$ has a fixed point, and $L$ and $J$ have a unique common fixed point. To see this, let $u_{0} \in V$. It follows from condition (1) that there exists $u_{1} \in V$ such that $L\left(u_{1}\right)=J\left(u_{0}\right)$. Repeating this process, we can find $\left\{u_{n}\right\} \subset V$ such that $L\left(u_{n}\right)=J\left(u_{n-1}\right)$ for $n \in \mathcal{N}$, which together with condition (3) yields

$$
\begin{aligned}
d_{b}\left(L\left(u_{n+1}\right), L\left(u_{n}\right)\right)= & d_{b}\left(J\left(u_{n}\right), J\left(u_{n-1}\right)\right) \\
\leq & \varphi d_{b}\left(L\left(u_{n}\right), L\left(u_{n+1}\right)\right)+\omega d_{b}\left(L\left(u_{n-1}\right), L\left(u_{n}\right)\right) \\
& +\eta d_{b}\left(L\left(u_{n-1}\right), L\left(u_{n+1}\right)\right)+\lambda d_{b}\left(L\left(u_{n}\right), L\left(u_{n-1}\right)\right) \\
\leq & \varphi d_{b}\left(L\left(u_{n}\right), L\left(u_{n+1}\right)\right)+\omega d_{b}\left(L\left(u_{n-1}\right), L\left(u_{n}\right)\right) \\
& +s \eta\left[d_{b}\left(L\left(u_{n-1}\right), L\left(u_{n}\right)\right)+d_{b}\left(L\left(u_{n}\right), L\left(u_{n+1}\right)\right)\right] \\
& +\lambda d_{b}\left(L\left(u_{n}\right), L\left(u_{n-1}\right)\right) .
\end{aligned}
$$

Then

$$
d_{b}\left(L\left(u_{n+1}\right), L\left(u_{n}\right)\right) \leq \frac{\omega+s \eta+\lambda}{1-\varphi-s \eta} d_{b}\left(L\left(u_{n}\right), L\left(u_{n-1}\right)\right) .
$$

Since $\frac{\omega+s \eta+\lambda}{1-\varphi-s \eta}<1$, we see that $\left\{L\left(u_{n}\right)\right\}$ is a Cauchy sequence. Moreover, by the completeness of the space, we deduce that $\lim _{n \rightarrow \infty} L\left(u_{n}\right)=r$ for some $r \in V$. From $L\left(u_{n}\right)=$ $J\left(u_{n-1}\right)$, it follows that $\lim _{n \rightarrow \infty} J\left(u_{n}\right)=r$. By condition $(2)$, we get

$$
L(r)=\lim _{n \rightarrow \infty} L\left(J\left(u_{n}\right)\right)=\lim _{n \rightarrow \infty} J\left(L\left(u_{n}\right)\right)=J(r),
$$


which yields $L(L(r))=L(J(r))=J(L(r))=J(J(r))$. By condition (3), we deduce that

$$
\begin{aligned}
d_{b}(J(r), J(J(r))) \leq & \varphi d_{b}(L(r), J(r))+\omega d_{b}(L(J(r)), J(J(r))) \\
& +\mu d_{b}(L(r), J(J(r)))+\eta d_{b}(L(J(r)), J(r))+\lambda d_{b}(L(r), L(J(r))) \\
= & \mu d_{b}(L(r), L(L(r)))+\eta d_{b}(L(r), L(L(r)))+\lambda d_{b}(L(r), L(L(r))),
\end{aligned}
$$

which implies $(1-\mu-\eta-\lambda) d_{b}(L(r), L(L(r))) \leq 0$. Since $1-\mu-\eta-\lambda>0$, we deduce that $L(r)$ is a common fixed point of $L$ and $J$.

For the uniqueness, if there exist $r, k \in V$ such that $L(r)=r=J(r)$ and $L(k)=k=$ $J(k)$, then by condition (3), it follows that

$$
\begin{aligned}
d_{b}(r, k)= & d_{b}(J(r), J(k)) \\
\leq & \varphi d_{b}(L(r), J(r))+\omega d_{b}(L(k), J(k)) \\
& +\mu d_{b}(L(r), J(k))+\eta d_{b}(L(k), J(r))+\lambda d_{b}(L(r), L(k) \\
= & \mu d_{b}(r, k)+\eta d_{b}(r, k)+\lambda d_{b}(r, k) .
\end{aligned}
$$

Clearly, we can get $(1-\mu-\eta-\lambda) d_{b}(r, k) \leq 0$. Since $1-\mu-\eta-\lambda>0$, we have $d_{b}(r, k)=0$ which implies $r=k$.

\section{Conclusions}

In this paper, we mainly study the existence and uniqueness of common fixed points for two mappings in complete $b$-metric spaces by virtue of the new functions $F$ and $\theta$, respectively. Moreover, we present two specific instances to show the availability of our results. Compared with the previous results, we weaken the conditions of the function $F$, which only needs to be non-decreasing. Hence, to some extent, our conclusions and methods improve the results of previous literature. However, for the application of the results, we need to continue to study, especially the existence of solutions of some integral equations and differential equations.

Author Contributions: L.C.: conceptualization, supervision; X.X.: investigation, writing-review and editing; Y.Z.: methodology; X.L.: writing-original draft. All authors read and approved the final manuscript.

Funding: This research was funded by Shandong Provincial Natural Science Foundation under grant ZR2020MA006 and the Introduction and Cultivation Project of Young and Innovative Talents in Universities of Shandong Province.

Institutional Review Board Statement: Not applicable.

Informed Consent Statement: Not applicable.

Data Availability Statement: All data included in this study are available upon request by contact with the corresponding author.

Acknowledgments: We would like to express our thanks to the anonymous referees and the editor for their constructive comments and suggestions, which greatly improved this article.

Conflicts of Interest: The authors declare no conflict of interest.

\section{References}

1. Czerwik, S. Contraction mappings in b-metric spaces. Acta Math. Inform. Univ. Ostravien. 1993, 1, 5-13.

2. Samet, B. The class of $(\varphi, \psi)$-type contractions in b-metric spaces and fixed point theorems. Fixed Point Theory Appl. $2015,2015,92$. [CrossRef]

3. Mohanta, S.K. Common fixed points in b-metric spaces endowed with a graph. Mat. Vesnik. 2016, 68, 140-154.

4. Imdad M.; Asim M.; Gubran R. Common fixed point theorems for g-generalized contractive mappings in $b$-metric spaces. Indian J. Math. 2018, 60, 85-105.

5. Wardowski, D. Fixed points of a new type of contractive mappings in complete metric spaces. Fixed Point Theory Appl. 2012, 2012, 94. [CrossRef] 
6. Cosentino, M.; Vetro, P. Fixed point results for F-contractive mappings of Hardy-Rogers-type. Filomat 2014, 28, 715-722. [CrossRef]

7. Hussain, N.; Salimi, P.S. Suzuki-Wardowski type fixed point theorems for $\alpha$-GF-contractions. Taiwan. J. Math. 2014, 18, 1879-1895. [CrossRef]

8. Lukács, A.; Kajántó, S. Fixed point theorems for various types of F-contractions in complete $b$-metric spaces. Fixed Point Theory 2018, 19, 321-334. [CrossRef]

9. Minak, G.; Helvac1, A.; Altun I. Ćirić type generalized F-contractions on complete metric spaces and fixed point results. Filomat 2014, 28, 1143-1151. [CrossRef]

10. Piri, H.; Kumam, P. Some fixed point theorems concerning F-contraction in complete metric spaces. Filomat 2014, 2014, 210. [CrossRef]

11. Goebel, K.; Reich, S. Uniform Convexity, Hyperbolic Geometry, and Nonexpansive Mappings; Marcel Dekker: New York, NY, USA, 1984.

12. Reich, S. Fixed points of contractive functions. Boll. Della Unione Mat. Ital. 1972, 5, 26-42.

13. Reich, S.; Shafrir, I. Nonexpansive iterations in hyperbolic spaces. Nonlinear Anal. 1990, 19, 537-558. [CrossRef]

14. Reich, S. Approximate selections, best approximations, fixed points, and invariant sets. J. Math. Anal. Appl. 1978, 62, 104-113. [CrossRef]

15. Hammad, H.A.; Bota, M.F.; Guran, L. Wardowski's contraction and fixed point technique for solving systems of functional and integral equations. J. Funct. Spaces 2021, 2021, 7017046. [CrossRef]

16. Hammad, H.A.; De la Sen, M.; Aydi, H. Analytical solution for differential and nonlinear integral equations via $F_{w_{e}}-S u z u k i$ contractions in $w_{e}$-modified-metric-like spaces. J. Funct. Spaces 2021, 2021, 6128586.

17. Hammad, H.A.; De la Sen, M.; Agarwal, P. New coincidence point results for generalized graph-preserving multivalued mappings with applications. Adv. Differ. Equ. 2021, 2021, 334. [CrossRef]

18. Suzuki, T. Fixed point theorems for single- and set-valued F-contractions in $b$-metric spaces. J. Fixed Point Theory Appl. 2018, 20, 35. [CrossRef]

19. Mirmostafaee, A.K. Fixed point theorems for set-valued mappings in $b$-metric spaces. Fixed Point Theory 2017, 18, 305-314. [CrossRef]

20. Jang, H.J. Some generalizations of fixed point theorems and common fixed point theorems. J. Fixed Point Theory Appl. 2018, 20,144 .

21. Chen, L.; Huang, S.; Li, C.; Zhao, Y. Several fixed-point theorems for F-contractions in complete Branciari $b$-metric spaces and applications. J. Funct. Spaces 2020, 2020, 7963242. [CrossRef]

22. Chifu, C.; Petruşel, G. Fixed points for multivalued contractions in b-metric spaces with applications to fractals. Taiwan. J. Math. 2014, 18, 1365-1375. [CrossRef]

23. Jungck, G. Commuting mappings and fixed points. Am. Math. Mon. 1976, 83, 261-263. [CrossRef]

24. Hardy, G.E.; Rogers, T. A generalization of a fixed point theorem of Reich. Can. Math. Bull. 1973, 16, 201-206. [CrossRef]

25. Sintunavarat, W. Nonlinear integral equations with new admissibility types in $b$-metric spaces. J. Fixed Point Theory Appl. 2016, 18, 397-416. [CrossRef] 